\title{
RECONOCIMIENTO DEL ESTADO EMOCIONAL MEDIANTE SEÑALES EEG
}

\author{
Rubén Marco, Eduardo Iáñez, Mario Ortiz, José M. Azorín \\ Brain-Machine Interface Systems Lab, Universidad Miguel Hernández de Elche \\ Avda. de la Universidad s/n, 03202 Elche, España \\ ruben.marco01@goumh.umh.es, eianez@umh.es, mortiz@umh.es,.jm.azorin@umh.es
}

\begin{abstract}
Resumen
En este trabajo se ha desarrollado un sistema BMI para reconocer el estado emocional de los sujetos mediante señales EEG. Se han definido tres estados emocionales diferentes: agradable, neutro y desagradable. Se ha diseñado un protocolo experimental que muestra a 5 sujetos diversas imágenes de cada uno de los estados planteados. Tras preprocesar las señales registradas se han obtenidos sus caracteristicas en el dominio de la frecuencia para posteriormente aplicar un clasificador SVM mediante validación cruzada para obtener los resultados. Se han evaluado 5 configuraciones de electrodos y dos tamaños de ventana de procesamiento. Los resultados obtenidos muestran que las señales registradas en las regiones próximas a las temporales son representativas del estado emocional y que la distinción entre estados agradables y neutros resulta más sencilla que el resto de clasificaciones, alcanzándose un porcentaje de acierto medio del $76 \%$ al respecto.
\end{abstract}

Palabras clave: BMI, EEG, estado emocional.

\section{INTRODUCCIÓN}

Las interfaces cerebro-máquina (BMIs, del inglés Brain-Machine Interfaces) son sistemas que, mediante el registro y el procesamiento de la actividad cerebral de una persona, permiten la comunicación entre sus funciones cognitivas y un dispositivo externo. Según su disposición, podemos distinguir entre dos tipos de BMIs: las invasivas (los electrodos se implantan directamente en el cerebro mediante cirugía) y las no invasivas (los electrodos se colocan sobre el cuero cabelludo). Dentro de estas últimas existen, además, dos grandes grupos: las evocadas (en las que es necesaria una estimulación externa para producir una respuesta en el sujeto) y las espontáneas (en las que no se requiere de ningún estímulo externo y es el usuario quien imagina las tareas por voluntad propia) [4].

Uno de los campos ampliamente estudiados dentro del ámbito de las BMIs es el del reconocimiento de emociones. En investigaciones anteriores se ha demostrado que el estudio de señales EEG resulta apropiado para realizar este tipo de tarea [7], y, además, se considera que las señales EEG son más fiables a la hora de analizar emociones que la expresión facial, gestual o corporal [6]. En cuanto a la clasificación de las emociones registradas, principalmente se distinguen dos modelos: los discretos, en los cuales las emociones se consideran estados independientes, y los dimensionales, en los que las emociones se distribuyen en un plano o espacio cuyos ejes se corresponden con los parámetros según los cuales serán clasificadas. Uno de los modelos discretos más utilizados es el que considera que cualquier emoción se puede asociar a una de las 6 denominadas básicas (ira, disgusto, miedo, alegría, tristeza y sorpresa), mientras que el modelo dimensional más extendido es el de evaluación-activación, que ubica las emociones en base a estos parámetros [1][3].

En los últimos años podemos encontrar diversos ejemplos de trabajos realizados en este ámbito. En [8] los sujetos fueron 5 niños de 5 años de edad, a los que mostraron expresiones faciales humanas que reflejaban estados de felicidad, tristeza, miedo o neutralidad. Las señales EEG fueron registradas de los lóbulos frontales con una frecuencia de muestreo de $250 \mathrm{~Hz}$ y se aplicaron filtros elípticos para conservar las bandas theta y alfa, mientras que para la clasificación se utilizó un modelo MLP de 2 capas con 10 neuronas en cada una.

En [11] las adquisición de las señales se realizó desde la base de datos DEAP, que contiene señales EEG pertenecientes a 32 personas que fueron registradas mientras éstas veían videos musicales, además de información facilitada por ellas mismas sobre las emociones que sintieron, siguiendo un modelo dimensional. Los autores dividieron estas señales en cuatro tipos e implementaron 4 clasificadores SVM binarios para realizar una diferenciación por pares.

En [2] se utilizó también la base de datos DEAP y un modelo dimensional de las emociones. Se realizó una reducción de la resolución de la señal 
y se aplicó un filtro paso banda para conservar la información perteneciente al rango de frecuencias de entre los 4 y los $45 \mathrm{~Hz}$, además de un algoritmo de eliminación de artefactos. En cuento a la clasificación, se implementó una SVM multiclase con kernel RBF para diferenciar entre los distintos conjuntos.

Este trabajo se centra en el diseño de una BMI no invasiva con el objetivo de diferenciar entre tres estados emocionales mediante señales EEG. Se mostrarán a los sujetos imágenes que evoquen estos estados para analizar como cambian sus señales cerebrales.

\section{MATERIAL Y MÉTODOS}

\subsection{EQUIPO DE REGISTRO}

Para registrar las señales EEG de cada individuo, se ha utilizado el equipo actiCHamp de la compañía Brain Vision LLC. Este equipo incluye 32 electrodos de registro $(31+1$ de referencia $)$ que son amplificados y discretizados con una resolución de 24 bits. La frecuencia de muestreo utilizada ha sido de $500 \mathrm{~Hz}$. Se ha utilizado Matlab tanto para registrar como para procesar los señales EEG. Mediante la API del equipo Matlab se ha conectado a software del actiCHamp, PyCorder (Brain Vision LLC), para obtener las señales EEG. Este software permite implementar un filtro paso banda con frecuencias de corte ajustables por el sujeto y un filtro Notch (o de rechazo de banda) para eliminar el ruido de la red eléctrica. Además, antes de realizar cada prueba se ha aplicado un electrolito en forma de gel electroconductor para reducir la impedancia entre la piel y el electrodo y obtener una buena calidad de señal. También se ha aplicado un gel abrasivo en el lóbulo de la oreja de los sujetos (donde se coloca el electrodo de referencia) para eliminar la grasa de la piel y aumentar la conductividad.

\subsection{PROTOCOLO EXPERIMENTAL}

Los estados emocionales que se han analizado en este trabajo son el agradable, el desagradable y el neutro. El primero de ellos se ha definido como un estado de alegría no necesariamente efusiva y más enfocada al bienestar. El estado desagradable se ha definido como disgusto focalizado en el rechazo o el asco. Y el estado neutro se ha definido como un estado de relajación sin ningún tipo de emoción asociada.

Para diferenciar entre estos tres estados emocionales, se ha utilizado un conjunto de fotografías escogido específicamente para cada individuo. La prueba se ha realizado individualmente con 5 sujetos varones y sanos de 23 años de edad, y se ha dividido en 3 fases:

- En primer lugar, se le ha realizado una pequeña entrevista a cada individuo para conocer el tipo de fotografías agradables y desagradables más propensas a evocarle las emociones deseadas.

- Tras la entrevista, se ha seleccionado un conjunto de 45 fotografías para cada sujeto (15 por cada estado emocional). Cada imagen ha sido mostrada durante 10 segundos mientras se registraban las señales EEG del sujeto. Durante la visualización, se ha instado a los voluntarios a que no realizasen movimientos musculares que pudiesen contaminar las señales registradas, y se han intercalado tiempos de descanso entre la muestra de las imágenes para que pudiesen parpadear. En la Figura 1 se muestra a un sujeto realizando esta fase del experimento.

- En las fotografías agradables aparecían familiares y amigos de los sujetos con expresión sonriente (el visionado de este tipo de imágenes evoca en los pacientes emociones similares, según estudios anteriores [9]).

- Las fotografías desagradables se correspondían con aquello que causaba un mayor rechazo a cada individuo (generalmente heridas en humanos).

- Las neutras mostraban paisajes homogéneos en blanco y negro.

- Por último, se ha realizado otra entrevista con cada individuo para conocer su feedback respecto a la prueba, de forma que si el sujeto considera que se han evocado en él las emociones deseadas, los datos se toman como válidos, y en caso contrario se descartan y se repite la segunda fase con un conjunto de imágenes distinto.

\subsection{REGISTRO}

El registro de datos se ha llevado a cabo mediante electroencefalograma con el equipo actiCHamp mientras los sujetos eran expuestos a los estímulos visuales. El software utilizado ha sido el programa PyCorder (con el que se han recogido los datos de los electrodos del gorro) y MATLAB (para almacenar y procesar los datos desde PyCorder). Se ha seguido una estrategia offline para obtener los datos, de forma que la clasificación de éstos tenía lugar una vez finalizada cada prueba. El conjunto de electrodos utilizado en el registro se muestra en la Figura 2. 


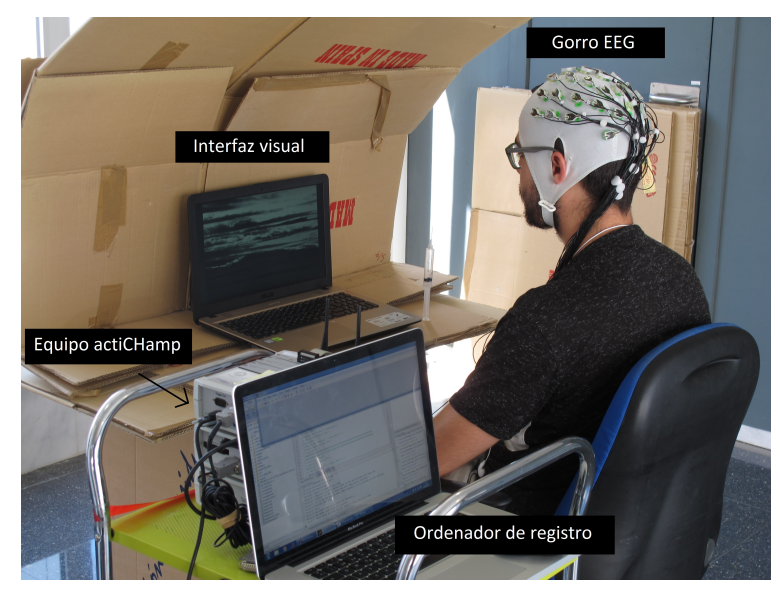

Figura 1: Usuario realizando las pruebas experimentales.

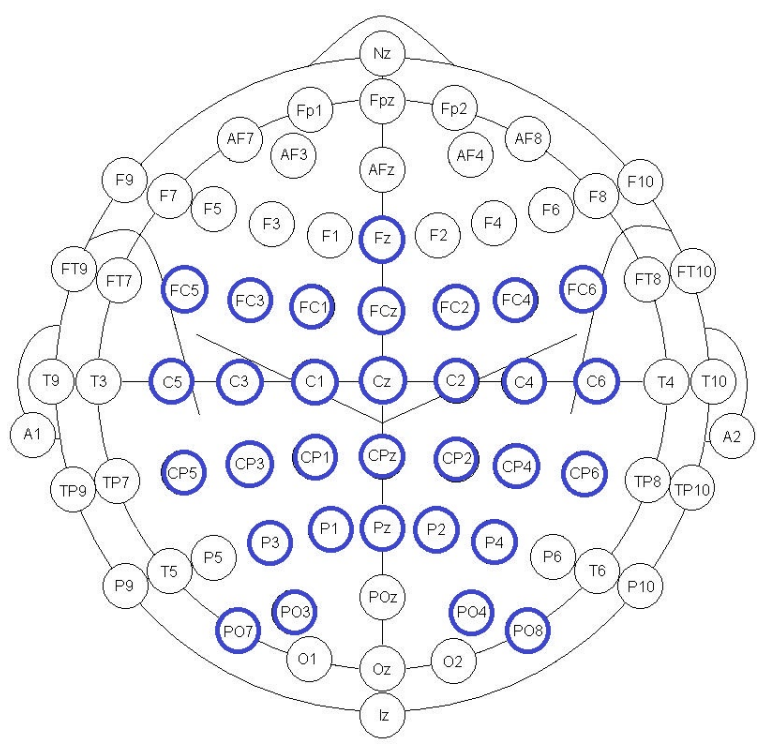

Figura 2: Conjunto de electrodos utilizado en el registro.

\subsection{PREPROCESADO}

Para reducir el ruido de las señales registradas, se han seguido dos procedimientos.

En primer lugar, el propio software de registro PyCoder implementa un filtro paso banda ajustable y un filtro Notch para eliminar la contaminación electromagnética en la señal. En este trabajo, el rango de frecuencias del filtro paso banda se ha establecido entre los 0.5 y los $100 \mathrm{~Hz}$, y el filtro Notch en $50 \mathrm{~Hz}$.

En segundo lugar, se ha aplicado un filtro de suavizado laplaciano con el objetivo de reducir la contaminación entre la señal obtenida por cada electrodo y la señal obtenida por el resto, de forma que en cada canal se almacene sólo la información que le corresponde. El filtro viene dado por la expresión (1), donde donde $d_{i j}$ representa la distancia entre el electrodo $i$ y el electrodo $j, V_{i}^{E R}$ y $V_{i}^{L A P}$ son los voltajes asociados al electrodo $i$ antes y después de la aplicación del filtro, respectivamente, y $S_{i}$ es el conjunto de los electrodos que rodean al electrodo $i$. Mientras que algunos trabajos se consideran como contaminantes sólo las señales de los electrodos circundantes [5], en éste se han tenido en cuenta, para cada electrodo, las del resto (al igual que en [10]). De esta forma, $S_{i}$ equivale a todo el conjunto de electrodos utilizado en el registro, a excepción del $i$.

$$
V_{i}^{L A P}=V_{i}^{E R}-\sum_{j \in S_{i}} \frac{1 / d_{i j}}{\sum_{j \in S_{i}}\left(1 / d_{i j}\right)} \cdot V_{j}^{E R}
$$

\subsection{EXTRACCIÓN DE CARACTERÍSTICAS}

Para mejorar la eficiencia del sistema, tras el preprocesado se han seleccionado sólo un subconjunto de electrodos para obtener sus características principales así como un rango de frecuencias.

En [7] se sugiere que hay una relación estrecha entre la actividad cerebral registrada en los lóbulos frontales y el estado emocional experimentado por una persona. En [11] se concuerda con ello y se añade que la actividad de las regiones centrales y temporales del cerebro también es apropiada para analizar emociones. Con esta información como referencia, se ha optado por escoger los datos registrados por distintos conjuntos de electrodos situados en las áreas frontal y central, principalmente, y en las zonas de éstas cercanas a la región temporal, como los electrodos FC5 y FC6. Tras la selección, los datos del resto de electrodos quedan descartados, y los siguientes 
procesos del sistema se realizan teniendo en cuenta únicamente los del subconjunto elegido.

En cuanto a la frecuencia, según [7], las ondas cerebrales en las que mejor se reflejan los cambios del estado emocional de una persona son las alfa. No obstante, en este caso parece no haber un consenso claro al respecto, puesto que otros estudios como [11] postulan que son las ondas beta las más apropiadas para analizar emociones. Ante esta disyuntiva, se ha tomado una resolución conservadora y se han analizado las señales con frecuencias en un rango de 8 a $35 \mathrm{~Hz}$, quedando así incluidas tanto la banda alfa como la beta. Para obtener información perteneciente a esta banda de frecuencia, se ha aplicado la transformada rápida de Fourier (mediante la función fft en MATLAB) a la señal de cada uno de los electrodos seleccionados, obteniendo así los vectores de características que, junto con sus etiquetas, han constituido la entrada al clasificador. Cabe destacar que cada vector de características no se corresponde con la totalidad de una tarea mental (los 10 segundos), sino que éstas se han dividido en ventanas de menor duración (con además, un solape del 50\% con la ventana anterior) para obtener un mayor número de vectores de características. Tener un mayor número de instancias de entrada a un clasificador es positivo y puede incrementar su eficacia, pero, por otra parte, dividir las tareas en muchas ventanas de muy corta duración redundaría en vectores de características de muy pocos elementos que podrían no ser suficientemente representativos de la tarea mental que realiza el sujeto. Por ello, se ha optado por elegir dos valores distintos de duración de las ventanas, de 1 y 2 segundos, y comparar los resultados obtenidos.

\subsection{CLASIFICACIÓN}

La última etapa del desarrollo del sistema consiste en el entrenamiento de un modelo de clasificación utilizando los datos de los vectores de características y su posterior utilización para clasificar datos de testeo que no hayan sido usados en la construcción del modelo, pudiendo así comprobar la eficacia del sistema.

Con el fin de obtener mejores resultados, se ha optado por realizar una diferenciación por pares. Para ello, se han entrenado tres modelos, uno de ellos con datos pertenecientes a los estados agradable y desagradable, otro con datos de los estados agradable y neutro, y otro con datos de los estados neutro y desagradable.

Para la creación de los distintos modelos de clasificación, se ha implementado una SVM (support vector machine) con función de base radial Gaussiana, dada por la expresión (2).

$$
K(u, v)=\exp \left(-\frac{\|u-v\|^{2}}{2 \sigma^{2}}\right)
$$

El testeo de cada uno de los modelos se ha realizado mediante validación cruzada, de forma que se han dividido los datos en 5 bloques y, en cada iteración, se ha entrenado el clasificador con 4 de ellos y se ha testeado con el restante. Las 5 iteraciones son promediadas para obtener el porcentajes de acierto. En la Figura 3 se muestra este proceso gráficamente.

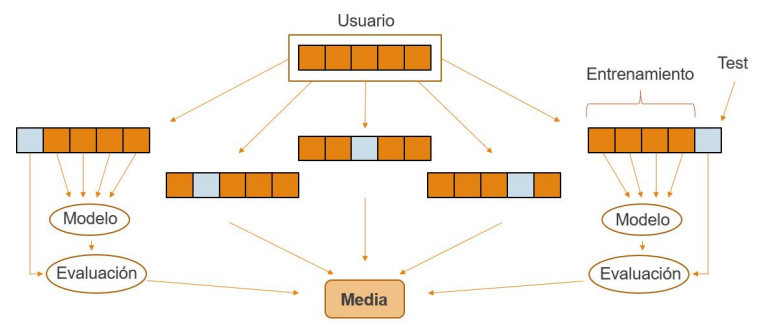

Figura 3: Esquema del método de validación cruzada utilizado.

\section{RESULTADOS}

En este apartado se exponen los diferentes resultados obtenidos al clasificar los datos tras probar distintas combinaciones del número de muestras por ventana y de selección de electrodos. Estos resultados reflejan el porcentaje de acierto de cada clasificación en cada usuario con respecto a la clasificación esperada, y se muestran en las tablas de la 1 a la 5, cada una de ellas correspondiente a una selección de electrodos distinta, dándose con la selección de electrodos de la tabla 1 una mayor influencia a la región frontal-central no cenital, con la selección de la tabla 2 un mayor peso al área más cenital, una mayor influencia a las regiones temporales en la selección de la tabla 3 y un peso distribuido en los casos de las tablas 4 y 5 . En estas tablas, cada fila se corresponde con uno de los usuarios y cada columna con un modelo de clasificación, representando las letras A, D y N, respectivamente, las tareas mentales agradables, desagradables y neutras (siendo cada modelo un clasificador entre dos de ellas).

Observando estas tablas de forma independiente, resulta llamativo el alto valor de la desviación estándar $(\sigma)$ que se obtiene entre los resultados de los usuarios en la mayoría de modelos. Esta es una de las principales dificultades a la hora de tratar de implementar un modelo común a 
Tabla 1: Resultados con selección de electrodos Fz, FC3, FC4, C5 y C6.

\begin{tabular}{|c|ccc|ccc|}
\cline { 2 - 7 } \multicolumn{1}{c|}{} & \multicolumn{3}{c|}{ Ventanas de 1 segundo } & \multicolumn{3}{c|}{ Ventanas de 2 segundos } \\
\cline { 2 - 7 } \multicolumn{1}{c|}{} & $\mathrm{A} / \mathrm{D}$ & $\mathrm{A} / \mathrm{N}$ & $\mathrm{N} / \mathrm{D}$ & $5 / \mathrm{D}$ & $\mathrm{A} / \mathrm{N}$ & $\mathrm{N} / \mathrm{D}$ \\
\hline Usuario 1 & $56,39 \%$ & $70,00 \%$ & $72,46 \%$ & $62,78 \%$ & $75,93 \%$ & $77,04 \%$ \\
Usuario 2 & $60,18 \%$ & $83,33 \%$ & $62,11 \%$ & $62,96 \%$ & $64,07 \%$ & $61,11 \%$ \\
Usuario 3 & $61,23 \%$ & $60,18 \%$ & $52,28 \%$ & $63,33 \%$ & $61,11 \%$ & $52,22 \%$ \\
Usuario 4 & $67,72 \%$ & $58,07 \%$ & $65,79 \%$ & $82,96 \%$ & $88,52 \%$ & $57,41 \%$ \\
Usuario 5 & $83,68 \%$ & $87,89 \%$ & $58,25 \%$ & $\mathbf{6} \%, 56 \%$ \\
\hline Media $\pm \sigma$ & $\mathbf{6 5 , 8 4} \pm 10,78 \%$ & $\mathbf{7 1 , 8 9} \pm 13,41 \%$ & $\mathbf{6 2 , 1 8} \pm 7,62 \%$ & $\mathbf{6 6 , 0 0} \pm 9,76 \%$ & $\mathbf{7 5 , 0 4} \pm 11,71 \%$ & $\mathbf{6 2 , 6 7} \pm 9,89 \%$ \\
\hline
\end{tabular}

Tabla 2: Resultados con selección de electrodos Fz, FC1, FC2, C1 y C2.

\begin{tabular}{|c|ccc|ccc|}
\cline { 2 - 7 } \multicolumn{1}{c|}{} & \multicolumn{3}{c|}{ Ventanas de 1 segundo } & \multicolumn{3}{c|}{ Ventanas de 2 segundos } \\
\cline { 2 - 7 } \multicolumn{1}{c|}{} & $\mathrm{A} / \mathrm{D}$ & $\mathrm{A} / \mathrm{N}$ & $\mathrm{N} / \mathrm{D}$ & $\mathrm{A} / \mathrm{D}$ & $\mathrm{A} / \mathrm{N}$ & $\mathrm{N} / \mathrm{D}$ \\
\hline Usuario 1 & $53,86 \%$ & $67,89 \%$ & $71,40 \%$ & $59,63 \%$ & $72,22 \%$ & $75,19 \%$ \\
Usuario 2 & $60,00 \%$ & $61,93 \%$ & $47,02 \%$ & $63,33 \%$ & $65,19 \%$ & $47,04 \%$ \\
Usuario 3 & $58,77 \%$ & $61,40 \%$ & $56,14 \%$ & $59,26 \%$ & $62,22 \%$ & $54,44 \%$ \\
Usuario 4 & $57,19 \%$ & $61,40 \%$ & $63,68 \%$ & $60,00 \%$ & $58,52 \%$ & $64,44 \%$ \\
Usuario 5 & $61,40 \%$ & $59,12 \%$ & $54,56 \%$ & $57,78 \%$ & $52,96 \%$ & $56,67 \%$ \\
\hline Media $\pm \sigma$ & $\mathbf{5 8 , 2 5} \pm 2,90 \%$ & $\mathbf{6 2 , 3 5} \pm 3,28 \%$ & $\mathbf{5 8 , 5 6} \pm 9,30 \%$ & $\mathbf{6 0 , 0 0} \pm 2,05 \%$ & $\mathbf{6 2 , 2 2} \pm 7,22 \%$ & $\mathbf{5 9 , 5 6} \pm 10,72 \%$ \\
\hline
\end{tabular}

Tabla 3: Resultados con selección de electrodos Fz, FC5, FC6, C5 y C6.

\begin{tabular}{|c|ccc|ccc|}
\cline { 2 - 7 } \multicolumn{1}{c|}{} & \multicolumn{3}{c|}{ Ventanas de 1 segundo } & \multicolumn{3}{c|}{ Ventanas de 2 segundos } \\
\cline { 2 - 7 } \multicolumn{1}{c|}{} & $\mathrm{A} / \mathrm{D}$ & $\mathrm{A} / \mathrm{N}$ & $\mathrm{N} / \mathrm{D}$ & $\mathrm{A} / \mathrm{D}$ & $\mathrm{A} / \mathrm{N}$ & $\mathrm{N} / \mathrm{D}$ \\
\hline Usuario 1 & $57,72 \%$ & $71,75 \%$ & $70,70 \%$ & $55,19 \%$ & $74,07 \%$ & $77,04 \%$ \\
Usuario 2 & $65,44 \%$ & $88,60 \%$ & $70,53 \%$ & $65,93 \%$ & $92,59 \%$ & $70,37 \%$ \\
Usuario 3 & $59,12 \%$ & $61,23 \%$ & $50,70 \%$ & $60,00 \%$ & $68,52 \%$ & $54,81 \%$ \\
Usuario 4 & $63,16 \%$ & $56,84 \%$ & $62,63 \%$ & $63,33 \%$ & $60,37 \%$ & $68,15 \%$ \\
Usuario 5 & $80,35 \%$ & $85,79 \%$ & $59,47 \%$ & $82,22 \%$ & $84,44 \%$ & $50,74 \%$ \\
\hline Media $\pm \sigma$ & $\mathbf{6 5 , 1 6} \pm 9,04 \%$ & $\mathbf{7 2 , 8 4} \pm 14,21 \%$ & $\mathbf{6 2 , 8 1} \pm 8,36 \%$ & $\mathbf{6 5 , 3 3} \pm 10,26 \%$ & $\mathbf{7 6 , 0 0} \pm 12,75 \%$ & $\mathbf{6 4 , 2 2} \pm 11,04 \%$ \\
\hline
\end{tabular}

Tabla 4: Resultados con selección de electrodos Fz, FC2, FC5, C1 y C6.

\begin{tabular}{|c|ccc|ccc|}
\cline { 2 - 7 } \multicolumn{1}{c|}{} & \multicolumn{3}{c|}{ Ventanas de 1 segundo } & \multicolumn{3}{c|}{ Ventanas de 2 segundos } \\
\cline { 2 - 7 } \multicolumn{1}{c|}{} & $\mathrm{A} / \mathrm{D}$ & $\mathrm{A} / \mathrm{N}$ & $\mathrm{N} / \mathrm{D}$ & $\mathrm{A} / \mathrm{D}$ & $\mathrm{A} / \mathrm{N}$ & $\mathrm{N} / \mathrm{D}$ \\
\hline Usuario 1 & $52,63 \%$ & $72,63 \%$ & $71,93 \%$ & $55,19 \%$ & $77,41 \%$ & $77,78 \%$ \\
Usuario 2 & $63,16 \%$ & $83,86 \%$ & $66,32 \%$ & $64,81 \%$ & $85,93 \%$ & $68,52 \%$ \\
Usuario 3 & $57,54 \%$ & $61,05 \%$ & $51,58 \%$ & $58,89 \%$ & $64,44 \%$ & $52,96 \%$ \\
Usuario 4 & $62,28 \%$ & $61,75 \%$ & $62,81 \%$ & $61,11 \%$ & $58,52 \%$ & $67,78 \%$ \\
Usuario 5 & $79,82 \%$ & $84,56 \%$ & $56,14 \%$ & $79,63 \%$ & $84,07 \%$ & $51,11 \%$ \\
\hline Media $\pm \sigma$ & $\mathbf{6 3 , 0 9} \pm 10,26 \%$ & $\mathbf{7 2 , 7 7} \pm 11,41 \%$ & $\mathbf{6 1 , 7 5} \pm 8,07 \%$ & $\mathbf{6 3 , 9 3} \pm 9,45 \%$ & $\mathbf{7 4 , 0 7} \pm 12,11 \%$ & $\mathbf{6 3 , 6 3} \pm 11,31 \%$ \\
\hline
\end{tabular}

Tabla 5: Resultados con selección de electrodos Fz, FC1, FC6, C2 y C5.

\begin{tabular}{|c|ccc|ccc|}
\cline { 2 - 7 } \multicolumn{1}{c|}{} & \multicolumn{3}{c|}{ Ventanas de 1 segundo } & \multicolumn{3}{c|}{ Ventanas de 2 segundos } \\
\cline { 2 - 7 } \multicolumn{1}{c|}{} & $\mathrm{A} / \mathrm{D}$ & $\mathrm{A} / \mathrm{N}$ & $\mathrm{N} / \mathrm{D}$ & $\mathrm{A} / \mathrm{D}$ & $\mathrm{A} / \mathrm{N}$ & $\mathrm{N} / \mathrm{D}$ \\
\hline Usuario 1 & $54,74 \%$ & $68,42 \%$ & $71,93 \%$ & $67,04 \%$ & $97,85 \%$ & $74,07 \%$ \\
Usuario 2 & $65,09 \%$ & $88,77 \%$ & $67,54 \%$ & $67,04 \%$ & $62,22 \%$ & $66,30 \%$ \\
Usuario 3 & $60,53 \%$ & $62,63 \%$ & $55,44 \%$ & $58,52 \%$ & $63,70 \%$ & $56,67 \%$ \\
Usuario 4 & $61,75 \%$ & $59,47 \%$ & $61,05 \%$ & $59,26 \%$ & $58,52 \%$ & $64,81 \%$ \\
Usuario 5 & $77,37 \%$ & $81,23 \%$ & $63,33 \%$ & $79,26 \%$ & $84,07 \%$ & $57,04 \%$ \\
\hline Media $\pm \sigma$ & $\mathbf{6 3 , 8 9} \pm 8,41 \%$ & $\mathbf{7 2 , 1 1} \pm 12,49 \%$ & $\mathbf{6 3 , 8 6} \pm 6,28 \%$ & $\mathbf{6 4 , 2 2} \pm 9,26 \%$ & $\mathbf{7 4 , 0 7} \pm 14,00 \%$ & $\mathbf{6 3 , 7 8} \pm 7,24 \%$ \\
\hline
\end{tabular}

todos los usuarios, y es que la actividad cerebral registrada varía significativamente entre estos y, con ello, los porcentajes de acierto en los distintos modelos: en la diferenciación entre dos tareas concretas, mientras que con algunos usuarios se ha obtenido un porcentaje de acierto bastante elevado (de hasta casi un 93\%), con otros este porcentaje ha resultado ser notablemente menor, y viceversa.

Para facilitar la comparación entre los resultados, en la 6 se muestra un resumen de estos donde cada celda se corresponde con la media de los porcentajes de acierto de todos los usuarios 
Tabla 6: Resumen de los resultados.

\begin{tabular}{|c|ccc|ccc|}
\hline \multirow{2}{*}{ Electrodos } & \multicolumn{3}{|c|}{ Ventanas de 1 segundo } & \multicolumn{3}{c|}{ Ventanas de 2 segundos } \\
\cline { 2 - 6 } & A/D & A/N & N/D & A/D & A/N & N/D \\
\hline Fz, FC3, FC4, C5, C6 & $65,84 \%$ & $71,89 \%$ & $62,18 \%$ & $66,00 \%$ & $75,04 \%$ & $62,67 \%$ \\
Fz, FC1, FC2, C1, C2 & $58,25 \%$ & $62,35 \%$ & $58,56 \%$ & $60,00 \%$ & $62,22 \%$ & $59,56 \%$ \\
Fz, FC5, FC6, C5, C6 & $65,16 \%$ & $72,84 \%$ & $62,81 \%$ & $65,33 \%$ & $76,00 \%$ & $64,22 \%$ \\
Fz, FC2, FC5, C1, C6 & $63,09 \%$ & $72,77 \%$ & $61,75 \%$ & $63,93 \%$ & $74,07 \%$ & $63,63 \%$ \\
Fz, FC1, FC6, C2, C5 & $63,89 \%$ & $72,11 \%$ & $63,86 \%$ & $64,22 \%$ & $74,07 \%$ & $63,78 \%$ \\
\hline
\end{tabular}

para un modelo de clasificación y una selección de electrodos. Observando esta tabla, parece evidente la influencia de las señales originadas en torno a las áreas temporales del cerebro en la detección de emociones, puesto que en las pruebas en las que se le ha dado más peso a la información registrada en estas regiones se han obtenido los porcentajes de acierto más elevados, y al anular esta influencia seleccionando un conjunto de electrodos cenital, el éxito de los clasificadores decae significativamente.

Respecto a la división en ventanas, se aprecian unos niveles mayores de acierto general de los clasificadores al dividir los datos en ventanas de 2 segundos de duración (equivalente a 1000 muestras) que al hacerlo en ventanas de 1 segundo. A pesar de que esta diferencia no es excesivamente acuciada, resulta de gran interés teniendo en cuenta que una división en ventanas de mayor duración reduce el número total de ventanas $\mathrm{y}$, por lo tanto, el número de vectores de características. Esto implica que los distintos modelos de clasificación se entrenan con un número de vectores y etiquetas menor, lo cual, si se tratase de conjuntos de datos similares, reduciría la eficacia de las predicciones posteriores. Aun así, los resultados mejoran al tomar ventanas de 2 segundos, sugiriendo que en los sujetos se registraron patrones relacionados con el estado emocional que se delimitan mejor en ventanas de esta duración.

Por otra parte, queda patente que con el modelo $\mathrm{A} / \mathrm{N}$ se obtiene un índice de acierto superior al de los otros dos en todos los casos. Esto es un indicio de que los registros correspondientes a estados agradables difieren de los neutros más que los desagradables, lo cual concuerda con los testimonios ofrecidos por los sujetos, puesto que, mientras que algunos aseguraban haber sentido el mismo impacto al visualizar los estímulos agradables y desagradables, otros confesaban que los del primer tipo habían repercutido en ellos más significativamente que los del segundo.

\section{CONCLUSIONES}

En este trabajo, se ha desarrollado una interfaz cerebro-máquina basada en señales EEG para reconocer el estado emocional de un conjunto de sujetos. Se han analizado tres estados emocionales: agradable, neutro y desagradable. El proceso se ha realizado desde la base, diseñando el protocolo de las pruebas, hasta la fase final de clasificación y obtención de resultados, pasando por las etapas de registro, procesado de datos y extracción de características.

A la luz de los resultados, se puede afirmar que, si bien los índices de acierto expuestos no son en general lo suficientemente elevados como para hablar de modelos de gran fiabilidad en sus predicciones, sí que presentan valores que permiten tildarlos de prometedores, especialmente en los casos de diferenciación de estados agradables frente a neutros (máxime teniendo en cuenta el reducido número de sujetos y de muestras, en comparación con otros estudios del mismo tipo).

Como trabajos futuros, repetir el estudio con un mayor número de usuarios permitiría obtener resultados más sólidos y extraer conclusiones más generales y mejor contrastadas. Deberá realizarse un análisis más detallado de las áreas del cerebro involucradas en el estado emocional del usuario. Además, un aumento del número de tareas por usuario redundaría en una mayor cantidad de vectores de características, lo cual mejoraría, previsiblemente, la eficacia de los clasificadores. Por otra parte, implementar un algoritmo de filtrado de artefactos permitiría a los usuarios expresar sus emociones de manera natural y fidedigna durante las pruebas, sin reprimir gestos o movimientos. Con todo ello, no resulta exagerado pensar que el sistema propuesto podría generar un modelo de clasificación común a todos los usuarios, que posteriormente podría utilizarse para realizar un reconocimiento a tiempo real del estado emocional de pacientes con dificultades para expresarlo de otra manera. 


\section{Agradecimientos}

El sistema inalámbrico de registro de señales EEG de 32 canales de Brain Products ha sido cofinanciado con fondos procedentes de la Unión Europea (P.O. FEDER 2007/2013), con la gestión de la Generalitat Valenciana. Se desea agradecer a los sujetos que han participado en los experimentos su colaboración altruista, indispensable para la realización del trabajo.

\section{English summary}

\section{EMOTIONAL STATE RECOGNITION THROUGH EEG SIGNALS}

\begin{abstract}
In this work, a BMI system has been developed to recognize the emotional state of the users through EEG signals. Three different emotional states have been defined (pleasant, neutral and unpleasant), as well as an experimental design to register them in 5 individuals, consisting on the visualization of distinct types of images for each of these states. After the register, the signals acquired have been filtered and a their features have been extracted in the frequency domain to apply a SVM classifier through cross validation, obtaining the results. 5 configurations of electrodes and two sizes of processing windows have been evaluated. The results show that signals registered near to temporal lobes are representative of the emotional state and that the distinction between pleasant and neutral states is easier than the rest of classifications, reaching an average success rate of $76 \%$.
\end{abstract}

Keywords: BMI, EEG, emotional state.

\section{Referencias}

[1] Abhang, P., Gawali, B., Mehrotra, S., (2016) Introduction to EEG and Speech-Based Emotion Recognition, Elsevier, London.

[2] Atkinson, J., Campos, D., (2016) "Improving BCI-based emotion recognition by combining
EEG feature selection and kernel classifiers", Expert Systems With Applications, pp. 35-41.

[3] Haq, S., Jackson, P.J.B., (2011) Machine Autidion: Principles, Algorithms and Systems, IGI Global, Hersey.

[4] Iáñez, E. (2012) Interfaces basadas en señales EEG y EOG para control de robots [tesis doctoral]. Universidad Miguel Hernández de Elche, Departamento de Ingeniería de Sistemas y Automática, Elche, España.

[5] McFarland, D.J., McCane, L.M., David, S.V., Wolpaw, J.R., (1997) "Spatial filter selection for EEG-based communication", Electroencephalography and clinical Neurophysiology, pp. 386-394.

[6] Naser, S.D., Saha, G., (2014) "Classification of emotions induced by music videos and correlation with participants' rating", Expert Systems with Applications, pp. 6057-6065.

[7] Niemic, P., Warren, K., (2002) "Studies of Emotion: A Theoretical and Emperical Review of Psychophysiological Studies of Emotion", Journal of Undergraduate Research, pp. 15-18.

[8] Othman, M., Wahab, A., Karim, I., Adawiah, M.D., Fakhri, I.T., (2013) "EEG emotion recognition based on the dimensional models of emotions", Procedia - Social and Behavioral Sciences, pp. 30-37.

[9] Pfeifer, J., Iacoboni, M., Mazziotta, J., Daprettoa, M., (2008) "Mirroring others' emotions relates to empathy and interpersonal competence in children", NeuroImage, pp. 2076-2085.

[10] Úbeda, A., Iáñez, E., Azorín, J.M., Pérez, C., (2013) "Endogenous Brain-Machine Interface based on the correlation of EEG maps", Computer Methods and Programs in Biomedicine, pp. 302-308.

[11] Zhang, Y., Ji, X., Zhang, S., (2016) "An approach to EEG-based emotion recognition using combined feature extraction method", Neuroscience Letters, pp. 152-157.

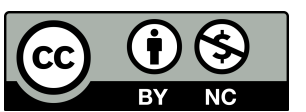

(c) 2018 by the authors. Submitted for possible open access publication under the terms and conditions of the Creative Commons Attribution CC-BY-NC 3.0 license (http://creativecommons.org/licenses/by-nc/3.0/). 